\title{
Discrete Signals, Feedback and Oscillation
}

\author{
Yoshifumi Okuyama \\ Humanitech Laboratory Co., Ltd. \\ (Professor Emeritus, Tottori University) \\ Tokushima, 770-8072 Japan \\ Email: oku@ humanitech-lab.jp \\ Telephone \& Fax: +81-88-6252545
}

\begin{abstract}
The problem of feedback and oscillation in animals and machines was once presented by $\mathbf{N}$. Wiener. Since then, the dynamcs of automatic (feedback) control and recent robotic systems has been examined based on this concept. In this paper, the stability of discrete (discrete-time and discrete-value) feedback systems is considered adapting to the computerized age. The stability condition of (nonlinear) discrete systems is derived by using the extended small-gain-theorem with an arbitrary number $q$. The relationships between this condition and Popov's criterion and Aizerman's conjecture in continuous signals are discussed. In numerical examples, some discrete time-responses and chaotic oscillations are shown in relation to the size of sampling-period and the magnitude of step-input.
\end{abstract}

\section{INTRODUCTION}

The problem of feedback and oscillation in animals and machines was once presented in [1]. Equation (4.52) in [1] corresponds to the sensitivity function (and return difference) in [3]. Furthermore, (4.53) is corresponding to the smallgain-theorem and the circle criterion in the recent control theory [11], [12]. In fact the concept of stability for feedback systems is already described in [1]. Although the content is time series in the statistics, the harmonic analysis of discrete or continuous signals is dealt with in [2]. With respect to discretetime signals, the analytical treatment of linear sampled-data feedback systems was developed in the 1950s-1960s [6], [7]. However, the theoretical analysis of discretized/quantized (computerized) control systems has not been elucidated. Only few results have been obtained, e.g.,[5], [8], [9].

In this paper, the stability of discrete-time and discretevalue (discretized) feedback systems is reconsidered [15], [16]. The relationships between this condition and Popov's criterion and Aizerman's conjecture in continuous signals are discussed. In numerical examples, some discrete timeresponses and chaotic oscillations are shown in relation to the size of sampling-period and the magnitude of step-input.

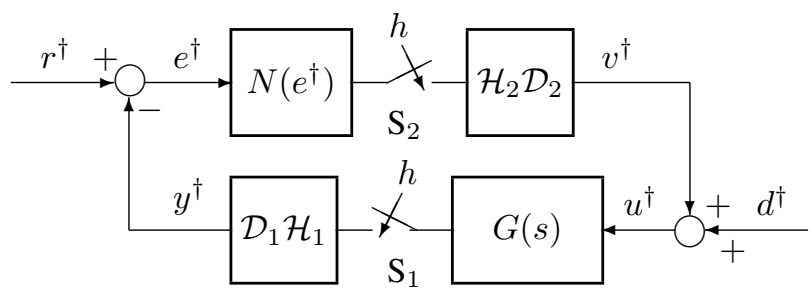

Fig. 1. Discretized nonlinear feedback system.

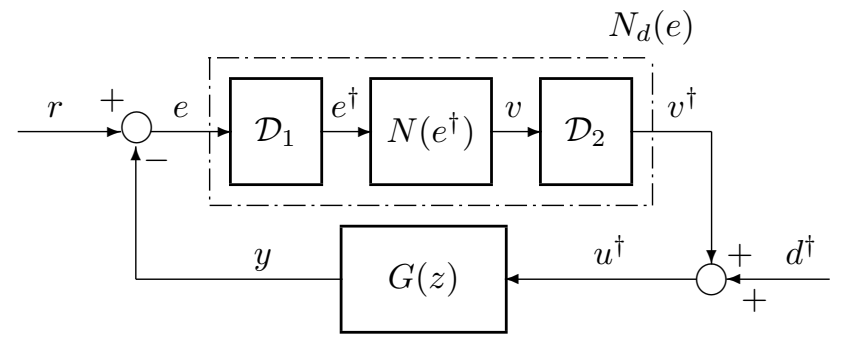

Fig. 2. Discrete nonlinear control system.

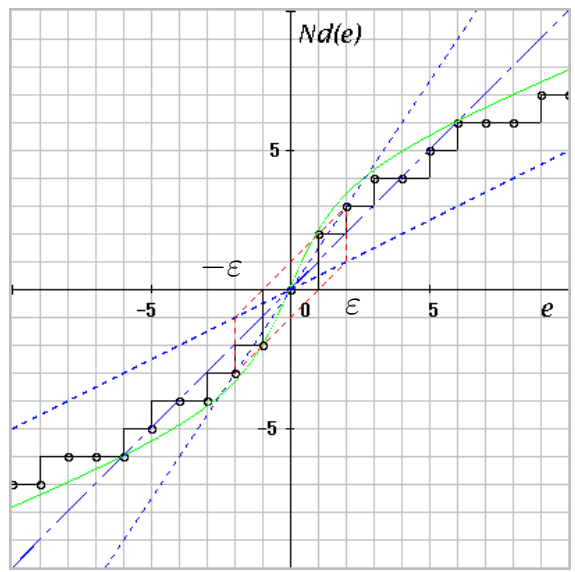

Fig. 3. An example of discretized nonlinear characteristic and stable sector.

\section{DiscRetized FeEdBACK System}

The discretized nonlinear feedback system in question is represented by a sampled-data control system as shown in Fig. 1. Here, $\mathcal{D}_{1} \mathcal{H}_{1}\left(\mathcal{H}_{2} \mathcal{D}_{2}\right)$ denotes the discretization and zero-order-hold, which are usually performed in $\mathrm{A} / \mathrm{D}(\mathrm{D} / \mathrm{A})$ conversion, and $G(s)$ is the transfer function of the linear controlled system. It is assumed that the two samplers with a sampling period $h$ operate synchronously. ${ }^{1}$ The sampleddata control system can be equivalently transformed into a discrete feedback system as shown in Fig. 2. Here, $G(z)$ is the $z$-transform of $G(s)$ together with zero-order-hold, and $\mathcal{D}_{1}$ and $\mathcal{D}_{2}$ are the discretizing units on the input and output sides of the nonlinear element, respectively. ${ }^{2}$ The relationship

\footnotetext{
${ }^{1}$ Of course, if nonlinear element $N(\cdot)$ is memoryless, sampler $S_{2}$ and holder $\mathcal{H}_{2}$ are not necessary.

${ }^{2}$ In any case, in the block diagrams, each variable, e.g., $e(k)$ should be considered a series of rectangular pulses with width $h$. Moreover, as a usual block-diagram expression, the transformed function $\hat{e}(z)$ should be also considered.
} 
between $e$ and $v^{\dagger}=N_{d}(e)$ in the figure becomes a stepwise (essentially point-to-point) nonlinear characteristic on integer grid coordinates as shown in Fig. 3. Here, the resolution value is chosen as 1.0 .

In Fig. 2, each symbol $e, u, y, \cdots$ indicates the sequence $e(k), u(k), y(k), \cdots,(k=0,1,2, \cdots)$ in discrete time, but for continuous value On the other hand, each symbol $e^{\dagger}, u^{\dagger}$, ... indicates a discrete value that can be assigned to an integer number, e.g.,

$$
\begin{aligned}
& e^{\dagger} \in\{\cdots,-3 \gamma,-2 \gamma,-\gamma, 0, \gamma, 2 \gamma, 3 \gamma, \cdots\}, \\
& u^{\dagger} \in\{\cdots,-3 \gamma,-2 \gamma,-\gamma, 0, \gamma, 2 \gamma, 3 \gamma, \cdots\},
\end{aligned}
$$

where $\gamma$ is the resolution of each variable. Here, it is assumed that the input and output signals of the nonlinear characteristic have the same resolution in the discretization. In the figure, $e^{\dagger}$ and $u^{\dagger}$ also represent the sequence $e^{\dagger}(k)$ and $u^{\dagger}(k)$. Without loss of generality, in this paper, $\gamma=1$ is assumed. Thus, the input and output variables of the nonlinear element can be considered in the set of integer numbers, i.e.,

$$
\begin{aligned}
& e^{\dagger}(k), u^{\dagger}(k) \in \mathbb{Z} \\
& \mathbb{Z}:=\{\cdots,-3,-2,-1,0,1,2,3, \cdots\} .
\end{aligned}
$$

\section{EQUIVALENT TRANSFORMATION}

In this paper, the stepwise and point-to-point nonlinear characteristic is partitioned into the following two sections:

$$
\begin{aligned}
& N_{d}(e)=K(e+\nu(e)), \quad 0<K<\infty, \\
& |\nu(e)| \leq \bar{\nu}<\infty,
\end{aligned}
$$

for $|e|<\varepsilon$, and

$$
\begin{aligned}
& N_{d}(e)=K(e+n(e)), \quad 0<K<\infty, \\
& |n(e)| \leq \alpha|e|, \quad 0<\alpha \leq 1,
\end{aligned}
$$

for $|e| \geq \varepsilon$, where $\nu(e)$ and $n(e)$ are nonlinear terms relative to nominal linear gain $K$. Equation (1) represents a bounded nonlinearity which exists in a finite region. On the other hand, (2) represents a sectorial nonlinearity of which the equivalent linear gain exists in a limited range. Therefore, when we consider the robust stability "in a global sense", it is sufficient to consider the nonlinear term $n(e)$. Here, $\varepsilon$ is a threshold of the input signal $e$. As a matter of course, (1) and (2) must be satisfied with respect to the discretized value $e=e^{\dagger}$ because $e^{\dagger} \in e$.

Based on the above consideration, the following new sequences $\bar{e}^{* \dagger}(k)$ and $\bar{w}^{* \dagger}(k)$ are defined:

$$
\begin{aligned}
& \bar{e}^{* \dagger}(k)=\bar{e}^{\dagger}(k)+q \cdot \frac{\Delta e^{\dagger}(k)}{h}, \\
& \bar{w}^{* \dagger}(k)=\bar{w}^{\dagger}(k)-\alpha q \cdot \frac{\Delta e^{\dagger}(k)}{h} .
\end{aligned}
$$

where $q$ is a non-negative number, $\bar{e}^{\dagger}(k)$ and $\bar{w}^{\dagger}(k)$ are neutral points of sequences $e^{\dagger}(k)$ and $w^{\dagger}(k)$,

$$
\begin{aligned}
& \bar{e}^{\dagger}(k)=\frac{e^{\dagger}(k)+e^{\dagger}(k-1)}{2}, \\
& \bar{w}^{\dagger}(k)=\frac{w^{\dagger}(k)+w^{\dagger}(k-1)}{2},
\end{aligned}
$$

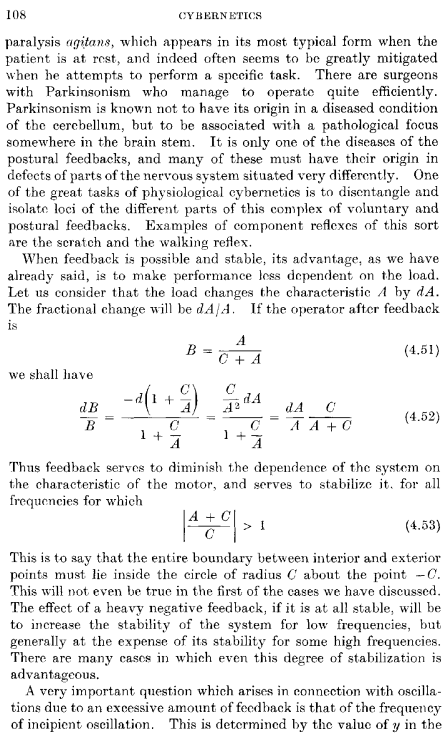

Fig. 4. Chapter 4 (page 108) in Cybernetics by N. Wiener.

and $\Delta e^{\dagger}(k)$ is the backward difference of sequence $e^{\dagger}(k)$,

$$
\Delta e^{\dagger}(k)=e^{\dagger}(k)-e^{\dagger}(k-1) .
$$

In this study the following assumption for the sequence is provided.

Assumption. The absolute value of the backward difference of sequence $e(k)$ does not exceed the resolution value $\gamma$, i.e.,

$$
|\Delta e(k)|=|e(k)-e(k-1)| \leq \gamma .
$$

If the assumption is satisfied, $\Delta e(k)$ becomes exactly $\pm \gamma$ or 0 because of the discretization $\mathcal{D}_{1}$.

The relationship between equations (3) and (4) in regard to the continuous values is shown by the block diagram in Fig. 5. In this figure, $\delta$ is defined as

$$
\delta(z):=\frac{2}{h} \cdot \frac{1-z^{-1}}{1+z^{-1}} .
$$

Equation (9) corresponds to the bilinear transformation between $z$ and $\delta$. Thus, the loop transfer function from $w^{*}$ to $e^{*}$ can be given by $F(\alpha, q, z)$, as shown in Fig. 6, where

$$
F(\alpha, q, z)=\frac{(1+q \delta(z)) K G(z)}{1+(1+\alpha q \delta(z)) K G(z)},
$$

and $r^{\prime}, d^{\prime}$ are transformed exogenous inputs. Here, the variables such as $w^{*}, u^{\prime}$ and $y^{\prime}$ written in Fig. 6 indicate the $z$-transformed ones. Obviously, when $q=0$, equation (10) becomes equivalent to the complementary sensitivity function of the nominal feedback system shown in Fig. 2, i.e.,

$$
S_{c}(z)=\frac{K G(z)}{1+K G(z)} .
$$

Note that the return difference and the sensitivity function of the nonmial system are given respectively as follows:

$$
R(z)=1+K G(z), \quad S(z)=1-S_{c}(z)=\frac{1}{1+K G(z)} .
$$




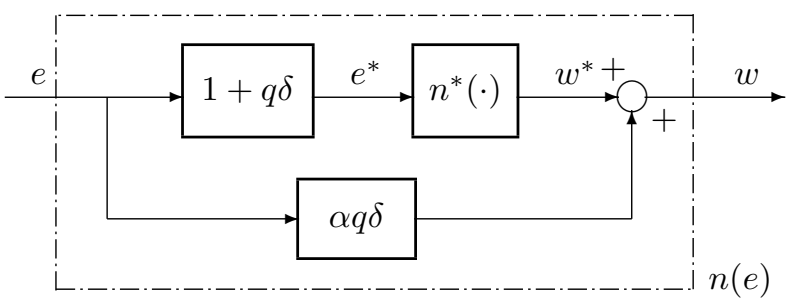

Fig. 5. Nonlinear subsystem.

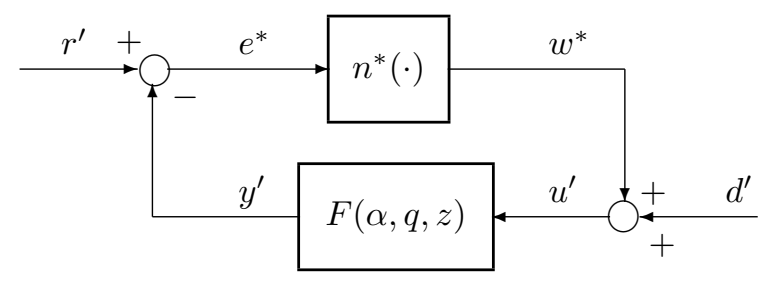

Fig. 6. Equivalent feedback system.

These concepts were presented in [1]. A copy of the book is shown in Fig. 4.

\section{TRAPEZOIDAl SUMmation}

In this section, some lemmas for norm inequalities are presented. Here, we define the following new nonlinear function:

$$
f(e):=n(e)+\alpha e .
$$

When considering the discretized output of the nonlinear term, $w^{\dagger}=n\left(e^{\dagger}\right)$, the following expression can be given:

$$
f\left(e^{\dagger}(k)\right)=w^{\dagger}(k)+\alpha e^{\dagger}(k) .
$$

From inequality (2), it can be seen that the function (13) belongs to the first and third quadrants. Considering the equivalent linear characteristic, the following inequality can be defined:

$$
0 \leq \vartheta(k):=\frac{f\left(e^{\dagger}(k)\right)}{e^{\dagger}(k)} \leq 2 \alpha .
$$

When this type of nonlinearity $\vartheta(k)$ is used, inequality (2) can be expressed as

$$
w^{\dagger}(k)=n\left(e^{\dagger}(k)\right)=(\vartheta(k)-\alpha) e^{\dagger}(k) .
$$

For the neutral points of $e^{\dagger}(k)$ and $w^{\dagger}(k)$, the following expression is given from (13):

$$
\frac{1}{2}\left(f\left(e^{\dagger}(k)\right)+f\left(e^{\dagger}(k-1)\right)\right)=\bar{w}^{\dagger}(k)+\alpha \bar{e}^{\dagger}(k) .
$$

Moreover, equation (15) is rewritten as

$$
\bar{w}^{\dagger}(k)=(\vartheta(k)-\alpha) \bar{e}^{\dagger}(k) .
$$

Since $\left|\bar{e}^{\dagger}(k)\right| \leq|\bar{e}(k)|$, the following inequality is satisfied when a round-down discretization is executed:

$$
\left|\bar{w}^{\dagger}(k)\right| \leq \alpha\left|\bar{e}^{\dagger}(k)\right| \leq \alpha|\bar{e}(k)| .
$$

Based on the above premise, the following norm inequalities are examined.
Lemma 1. The following inequality holds for a positive integer $p$ :

$$
\left\|\bar{w}^{\dagger}(k)\right\|_{2, p} \leq \alpha\left\|\bar{e}^{\dagger}(k)\right\|_{2, p} \leq \alpha\|\bar{e}(k)\|_{2, p} .
$$

Here, $\|\cdot\|_{2, p}$ denotes the Euclidean norm, which can be defined by

$$
\|x(k)\|_{2, p}:=\left(\sum_{k=1}^{p} x^{2}(k)\right)^{1 / 2} .
$$

Proof. The proof is clear from inequality (17).

Lemma 2. If the following inequality is satisfied in regard to the inner product of the neutral points of (13) and the backward difference (7):

$$
\left\langle\bar{w}^{\dagger}(k)+\alpha \bar{e}^{\dagger}(k), \Delta e^{\dagger}(k)\right\rangle_{p} \geq 0,
$$

the following inequality can be obtained:

$$
\left\|\bar{w}^{* \dagger}(k)\right\|_{2, p} \leq \alpha\left\|\bar{e}^{* \dagger}(k)\right\|_{2, p}
$$

for any $q \geq 0$. Here, $\langle\cdot, \cdot\rangle_{p}$ denotes the inner product, which can be defined as

$$
\left\langle x_{1}(k), x_{2}(k)\right\rangle_{p}=\sum_{k=1}^{p} x_{1}(k) x_{2}(k) .
$$

Proof. The proof is given in [14], [17].

Inequality (20) is satisfied by using the left inequality of (18). With respect to the input of $n^{*}(\cdot)$, the following can be obtained from the second inequality of (18):

$$
\left\|\bar{w}^{* \dagger}(k)\right\|_{2, p} \leq \alpha\left\|\bar{e}^{*}(k)\right\|_{2, p},
$$

when inequality (19) is satisfied.

The left side of inequality (19) can be expressed as a sum of trapezoidal areas.

Lemma 3. For any step $p$, the following equation is satisfied:

$$
\begin{aligned}
& \sigma(p):=\left\langle\bar{w}^{\dagger}(k)+\alpha \bar{e}^{\dagger}(k), \Delta e^{\dagger}(k)\right\rangle_{p} \\
& =\frac{1}{2} \sum_{k=1}^{p}\left(f\left(e^{\dagger}(k)\right)+f\left(e^{\dagger}(k-1)\right)\right) \Delta e^{\dagger}(k) .
\end{aligned}
$$

Proof. The proof is clear from (16)

Figures 7 (a) and (b) show examples of the sum of trapezoidal areas for $f(e)$, when $e$ is a sinusoidal input with amplitude 9.5, i.e., $e(k)=9.5 \sin \omega k$ ( $\omega$ :an arbitrary number).

(a) The sinusoid starts from 0 to 9.0 , and then decreases to 5.0.

(b) The sinusoid starts from 0 , passes 9.0, 0.0, -9.0, and increases to -5.0 .

In any case, the sum of trapezoids will be cancelled.

In general, the sum of trapezoidal areas holds the following property.

Lemma 4. If assumption (8) is satisfied in regard to the discretization of the control system, the sum of trapezoidal areas becomes non-negative for any $p$, that is,

$$
\sigma(p) \geq 0 .
$$




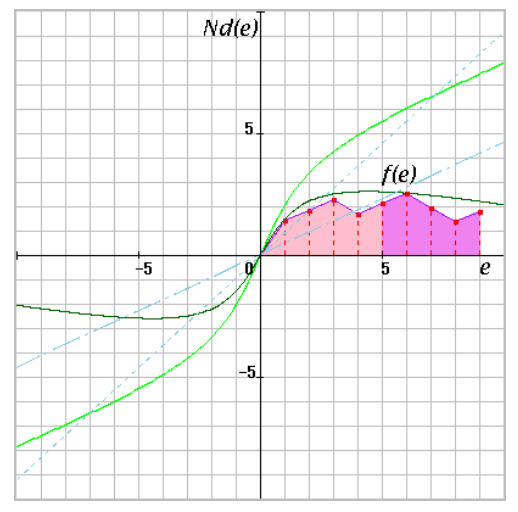

(a)

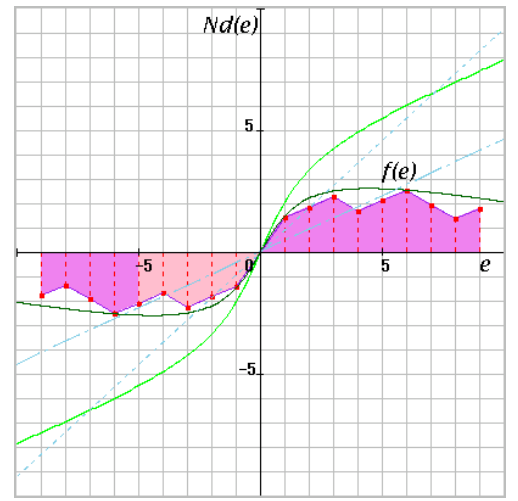

(b)

Fig. 7. Non-negative characteristics of trapezoidal summation.

Proof. Since $f\left(e^{\dagger}(k)\right)$ belongs to the first and third quadrants, the area of each trapezoid

$$
\tau(k):=\frac{1}{2}\left(f\left(e^{\dagger}(k)\right)+f\left(e^{\dagger}(k-1)\right)\right) \Delta e^{\dagger}(k)
$$

is non-negative when $e(k)$ increases (decreases) in the first (third) quadrant. On the other hand, the trapezoidal area $\tau(k)$ is non-positive when $e(k)$ decreases (increases) in the first (third) quadrant. The sum of trapezoidal area is given from (22) as:

$$
\sigma(p)=\sum_{k=1}^{p} \tau(k)
$$

Therefore, the following result is derived based on the above. The sum of trapezoidal areas becomes non-negative, $\sigma(p) \geq 0$, regardless of whether $e(k)$ (and $e^{\dagger}(\mathrm{k})$ ) increases or decreases. The detailed explanation of the proof is given in [14], [17].

\section{Stability Condition}

From the equivalent loop characteristic as shown in Fig. 6, the following inequality can be given for $z=\mathrm{e}^{j \omega h}$ :

$$
\begin{aligned}
& \left\|\bar{e}^{*}(z)\right\|_{2, p} \leq c_{1}\left\|\bar{r}^{\prime}(z)\right\|_{2, p}+c_{2}\left\|\bar{d}^{\prime}(z)\right\|_{2, p} \\
& +\sup _{z=1}|F(\alpha, q, z)| \cdot\left\|\bar{w}^{* \dagger}(z)\right\|_{2, p} .
\end{aligned}
$$

Here, $\bar{r}^{\prime}(z)$ and $\bar{d}^{\prime}(z)$ denote the $z$-trafor the neutral points of sequences $r^{\prime}(k)$ and $d^{\prime}(k)$, respectively. Moreover, $c_{1}$ and $c_{2}$ are positive constants. tained:

By using inequality (21), the following expression is ob-

$$
\begin{aligned}
& \left(1-\alpha \cdot \sup _{z=1}|F(\alpha, q, z)|\right)\left\|\bar{e}^{*}(z)\right\|_{2, p} \\
& \leq c_{1}\left\|\bar{r}^{\prime}(z)\right\|_{2, p}+c_{2}\left\|\bar{d}^{\prime}(z)\right\|_{2, p} .
\end{aligned}
$$

Therefore, if the following inequality (i.e., the small gain theorem with respect to $\ell_{2}$ gains) is valid:

$$
\left|F\left(\alpha, q, \mathrm{e}^{j \omega h}\right)\right| \leq 1 / \alpha,
$$

the sequences $\bar{e}^{*}(k), \bar{e}(k), e(k)$ and $y(k)$ in the feedback system are restricted in finite values when exogenous inputs $r(k), d(k)$ are finite and $p \rightarrow \infty$.

By applying the small gain theorem to the transformed loop characteristic with an arbitrary non-negative number $q$, the robust stability condition of the discrete nonlinear feedback system is derived based on (8).

Theorem 1. If there exists a $q \geq 0$ in which the sector parameter $\alpha$ in regard to nonlinear term $n(\cdot)$ satisfies the following inequality, the discrete-time control system with sector nonlinearity (2) is robust stable in an $\ell_{2}$ sense:

$$
\begin{aligned}
& \alpha<\eta(q, \omega):= \\
& \frac{-q \Omega V+\sqrt{q^{2} \Omega^{2} V^{2}+\left(U^{2}+V^{2}\right)\left\{(1+U)^{2}+V^{2}\right\}}}{U^{2}+V^{2}}, \\
& \forall \omega \in\left[0, \omega_{c}\right], \quad \omega_{c}: \text { cutoff frequency }
\end{aligned}
$$

when the linearized system with nominal gain $K$ is stable. Here, $\Omega(\omega)$ is the distorted frequency of angular frequency $\omega$ and is given by

$$
\delta\left(\mathrm{e}^{j \omega h}\right)=j \Omega(\omega)=j \frac{2}{h} \tan \left(\frac{\omega h}{2}\right), \quad j=\sqrt{-1} .
$$

In addition, $U(\omega)$ and $V(\omega)$ are the real and the imaginary parts of $K G\left(\mathrm{e}^{j \omega h}\right)$, respectively.

Proof. By substituting (10) into inequality (28), the following is obtained:

$$
\left|\frac{(1+j q \Omega(\omega)) K G\left(\mathrm{e}^{j \omega h}\right)}{1+(1+j \alpha q \Omega(\omega)) K G\left(\mathrm{e}^{j \omega h}\right)}\right|<\frac{1}{\alpha} .
$$

From the square of both sides of inequality (31),

$\alpha^{2}\left(1+q^{2} \Omega^{2}\right)\left(U^{2}+V^{2}\right)<(1+U-\alpha q \Omega V)^{2}+(V+\alpha q \Omega U)^{2}$

Then,

$$
\alpha^{2}\left(U^{2}+V^{2}\right)+2 \alpha q \Omega V-\left\{\left(1+U^{2}\right)+V^{2}\right\}<0 .
$$

Consequently, as a solution of inequality (32),

$$
\alpha<\frac{-q \Omega V+\sqrt{q^{2} \Omega^{2} V^{2}+\left(U^{2}+V^{2}\right)\left\{(1+U)^{2}+V^{2}\right\}}}{U^{2}+V^{2}}
$$

can be given.

Since inequality (29) in Theorem 1 is for all $\omega$ (and $\Omega$ ) considered and a certain $q$, the condition is rewritten as the following max-min problem.

Corollary. If the following inequality is satisfied, the discretetime control system with sector nonlinearity (2) is robust stable:

$$
\alpha<\eta\left(q_{0}, \omega_{0}\right)=\max _{q} \min _{\omega} \eta(q, \omega),
$$


when the linearized system with nominal gain is stable

Naturally, the stability condition becomes that of continuous-time and continuous-value nonlinear control systems, when the sampling period $h$ and the resolution $\gamma$ approach zero. Inequality (29) in Theorem 1 corresponds to Popov's criterion for discrete-time systems and contains the circle criterion for nonlinear time-varying (discrete-time) systems in a special case. The relationship between them will be described in the next section.

\section{RELATION TO POPOV's CRITERION}

Inequality (31) can be rewritten as follows:

$$
\left|\frac{\alpha H\left(\alpha, q, \mathrm{e}^{j \omega h}\right)}{1+\alpha H\left(\alpha, q, \mathrm{e}^{j \omega h}\right)}\right|<1,
$$

where

$$
H\left(\alpha, q, \mathrm{e}^{j \omega h}\right)=\frac{(1+j q \Omega(\omega)) K G\left(\mathrm{e}^{j \omega h}\right)}{1+(1-\alpha) K G\left(\mathrm{e}^{j \omega h}\right)} .
$$

From (34), the following inequality is obtained:

$$
2 \alpha \cdot \Re\left\{H\left(\alpha,, q, \mathrm{e}^{j \omega h}\right)\right\}+1>0 .
$$

Therefore, the following robust stability condition can be given:

$$
\Re\left\{\frac{1+(1+\alpha) K G\left(\mathrm{e}^{j \omega h}\right)+2 j \alpha q \Omega(\omega) K G\left(\mathrm{e}^{j \omega h}\right)}{1+(1-\alpha) K G\left(\mathrm{e}^{j \omega h}\right)}\right\}>0,
$$

which is equivalent to inequality (29). When $\alpha=1$ is chosen, inequality (36) can be written as follows:

$$
\frac{1}{2 K}+\Re\left\{(1+j q \Omega(\omega)) G\left(\mathrm{e}^{j \omega h}\right)\right\}>0 .
$$

In this case, the allowable sector of nonlinear characteristic $N(\cdot)$ is given as

$$
0 \leq N(e) e \leq 2 K e^{2}, \quad e \neq 0
$$

When $h$ approaches zero (or $\omega$ is a low frequency), inequalities (37) and (38) are equivalent to an expression of Popov's criterion for continuous-time systems.

In case of $q=0$, the left side of (29) becomes the inverse of the absolute value of complementary sensitivity function (11) for $z=\mathrm{e}^{j \omega h}$.

$$
\eta(0, \omega)=\frac{\sqrt{\left.\left(1+U^{2}\right)\right)+V^{2}}}{\sqrt{U^{2}+V^{2}}}=\frac{1}{\left|S_{c}\left(\mathrm{e}^{j \omega h}\right)\right|}>\alpha .
$$

On the other hand, from (36)

$$
\Re\left\{\frac{1+(1+\alpha) K G\left(\mathrm{e}^{j \omega h}\right)}{1+(1-\alpha) K G\left(\left(\mathrm{e}^{j \omega h}\right)\right.}\right\}>0
$$

is obtained. Inequalities (39) and (40) correspond to the circle criterion for nonlinear time-varying systems.

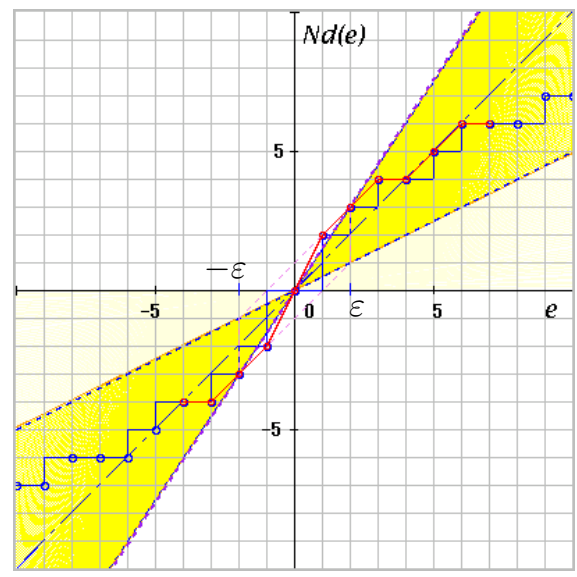

Fig. 8. Discretized nonlinear characteristic and stable sector for Example-1.

\section{VALIDITY OF AIZERMAN's CONJECTURE}

In the following case, Theorem-1 becomes equal to the robust stability condition of the linear interval gain that corresponds to Aizerman's conjecture which was extended into discrete-time systems.

Theorem 2. If the right side of (33) is satisfied at the saddle point,

$$
\left(\frac{\partial \eta(q, \omega)}{\partial q}\right)_{q=q_{0}, \omega=\omega_{0}}=0
$$

inequality (29) of Theorem-1 becomes equal to the robust stability condition provided for a linear time-invariant discretetime system.

Proof. This theorem can easily be proven by using the right side of (29). Then,

$$
\frac{\partial \eta(q, \omega)}{\partial q}=\frac{-\eta(q, \omega) \Omega(\omega) V(\omega)}{\sqrt{q^{2} \Omega^{2} v^{2}+\left(U^{2}+V^{2}\right)\left\{(1+U)^{2}+V^{2}\right\}}} .
$$

From (41), the following can be obtained:

$$
\eta\left(q_{0}, \omega_{0}\right) \Omega\left(\omega_{0}\right) V\left(\omega_{0}\right)=0 .
$$

Obviously, $\eta(q, \omega)>0$. Moreover, since $0<\omega_{0}<\pi / h$, $\Omega\left(\omega_{0}\right)>0$ from (30). Then,

$$
V\left(\omega_{0}\right)=0
$$

is obtained. Thus,

$$
\eta\left(q_{0}, \omega_{0}\right)=\frac{\left|1+U\left(\omega_{0}\right)\right|}{\left|U\left(\omega_{0}\right)\right|}>\alpha
$$

Inequality (45) corresponds to the stability condition which was determined for the time-invariant discrete-time system with a linear gain, i.e., the Nyquist stability condition for a discrete-time system.

Theorem 2 shows that the robust stability condition for a linear time-invariant system (the concept of interval set) can be applied to nonlinear discrete-time control systems, when (41) is satisfied. However, (33) is not always valid at the saddle point iven in (41). In the following example, it can be shown that there are counter examples of Aizerman's conjecture extended into the nonlinear discrete-time systems. 


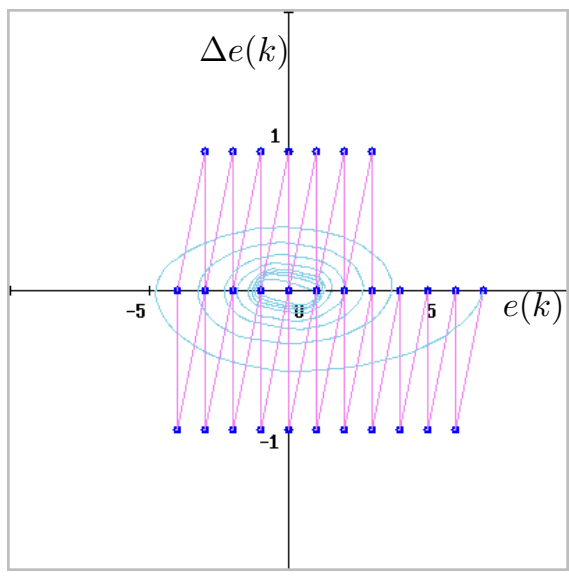

Fig. 9. Phase trace $(e(k), \Delta e(k))$ when 0.077 and $r=0.2$.

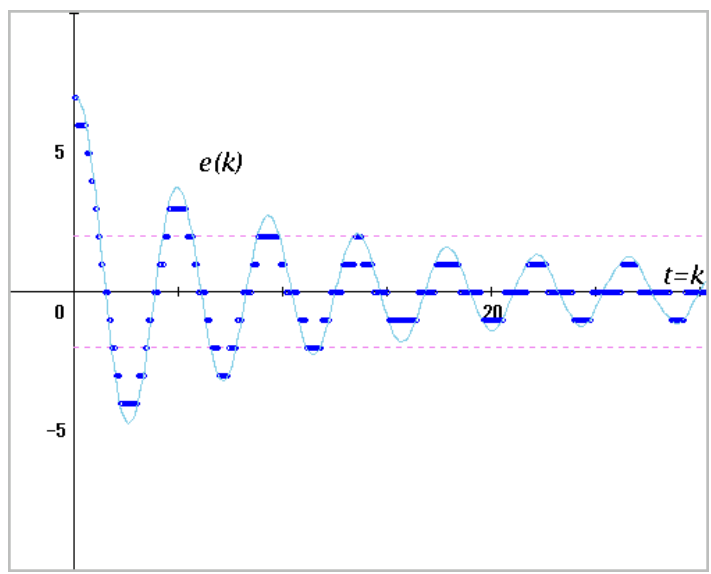

Fig. 10. Step response $e(k), e^{\dagger}(k)$, when $h=0.077$ and $r=0.7$.

\section{NUMERICAL EXAMPLES}

Example 1. Consider the following continuous system:

$$
G(s)=\frac{K_{p}(s+5)}{s(s+1)(s+2)}, \quad K_{p}=1.0 .
$$

Here, the same nonlinear characteristic is chosen as shown in Fig. 8. When the threshold $\varepsilon=2.0$ is specified, the sectorial area of the stepwise nonlinearity for $\varepsilon \leq|e|<35.0$ can be determined as $[0.5,1.5]$.

In this example, the sampling period is chosen as $h=$ 0.077 . The max-min value can be calculated as follows:

$$
\max _{q} \eta\left(q, \omega_{0}\right)=\eta\left(q_{0}, \omega_{0}\right)=0.5,
$$

when the nominal gain $K=1.0$. Hence, inequalities (29) and (33) are given by $\alpha<0.5$, and the stable area is determined as $[0.5,1.5]$. This sector agrees approximately with the above area as shown in Fig. 8. Since the stability region of control systems with linear gain $K$ is given as $0<K<1.5$, the discrete nonlinear feedback system in question is valid for Aizerman's conjecture extended into the nonlinear discrete-time systems.

Figures 9 and 10 show a phase trace ${ }^{3}$ and a step response

\footnotetext{
${ }^{3}$ When $h \rightarrow 0$, the phase traces correspond to trajectories in the phase plane for a continuous system.
}

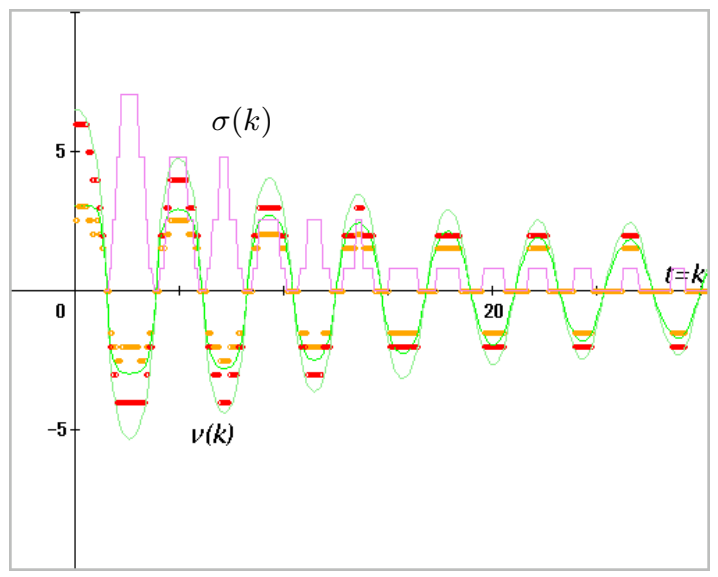

Fig. 11. Output signals $v(k), v^{\dagger}(k)$, and trapezoidal summation $\sigma(k)$.

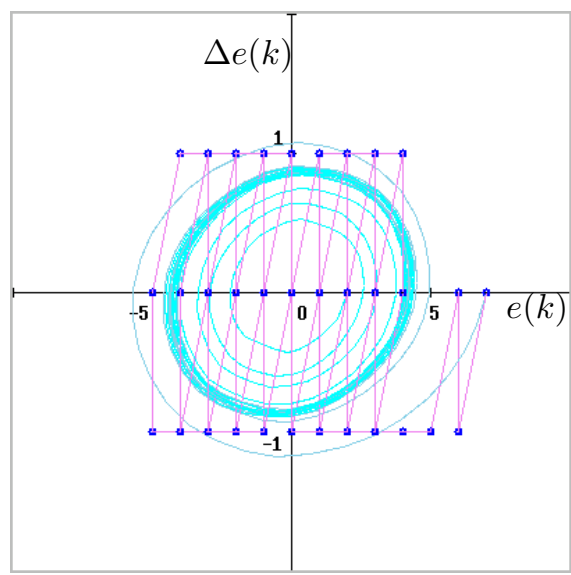

Fig. 12. Phase traces $(e(k), \Delta e(k))$ when $h=0.15$ and $r=2.0,7.0$.

for $e(k)$ (and $e^{\dagger}(k)$ ). As shown "small circles" in Fig. 9, the differences of discretized signals, $\Delta e^{\dagger}(k)$, are traced only on \pm 1 or 0 . In this case, phase trace $(e(k), \Delta e(k))$ with respect to continuous-value output $y(k)$ converges to a (pseudo) limit cycle as shown in Fig. 9. Figure 11 shows the output sequences of nonlinear element $f(\cdot)$ and the sum of trapezoidal areas $\sigma(k)$.

On the other hand, when a larger sampling period, e.g,, $h=0.15$ is chosen, a belt-like limit cycle (chaotic oscillation) may occur as shown in Fig. 12. In this case, step responses for $e(k)$ (and $e^{\dagger}(k)$ ) is shown in Fig. 13. The output sequences of nonlinear element $f(\cdot)$ and the sum of trapezoidal areas $\sigma(k)$ are calculated as shown in Fig. 14. Figure 15 shows $\Delta e^{\dagger}(k)$ when the sampling period $h$ increases from 0.01 to 0.75 . Obviously, assumption (8) is satisfied for at least $h \leq$ 0.15 . Of course, the valid region and chaotic behaviors will be dependent on the magnitude of input $r$.

Example 2. Consider the following controlled system:

$$
G(s)=\frac{K_{p}(-s+8)(s+4)}{s(s+0.2)(s+16)}, \quad K_{p}=1.0 .
$$

The same nonlinear characteristic is chosen as shown in Fig. 8 for Example 1. When the threshold $\varepsilon=2.0$ is specified, the sectorial area of the stepwise nonlinearity for $\varepsilon \leq|e|<35.0$ 


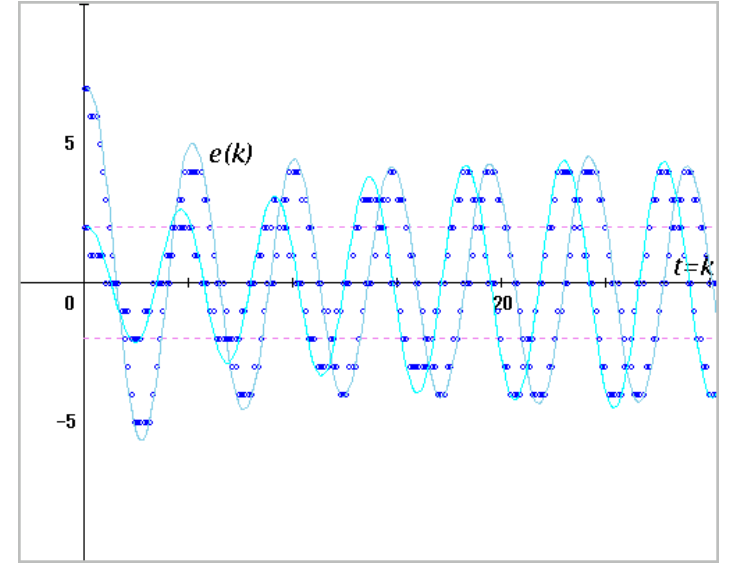

Fig. 13. Step responses $e(k)$ and $e^{\dagger}(k)$ when $h=0.077, r=0.2$ and $r=0.7$.

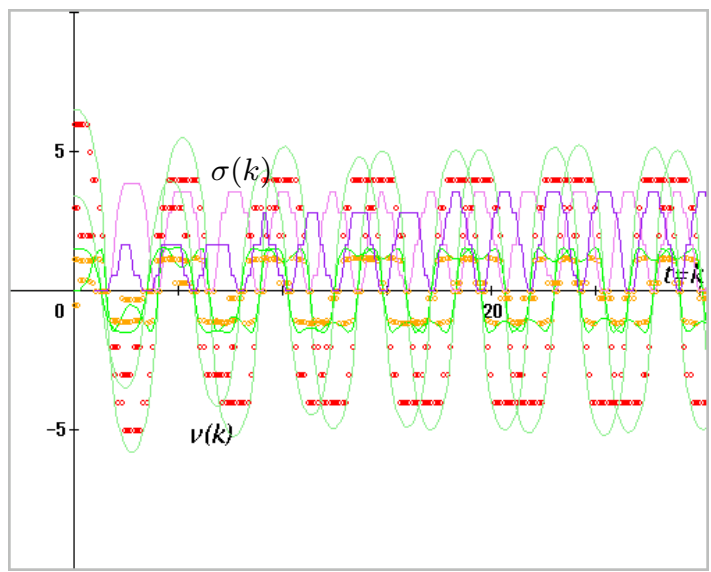

Fig. 14. Output signals $v(k)$ and trapezoidal summation $\sigma(k)$.

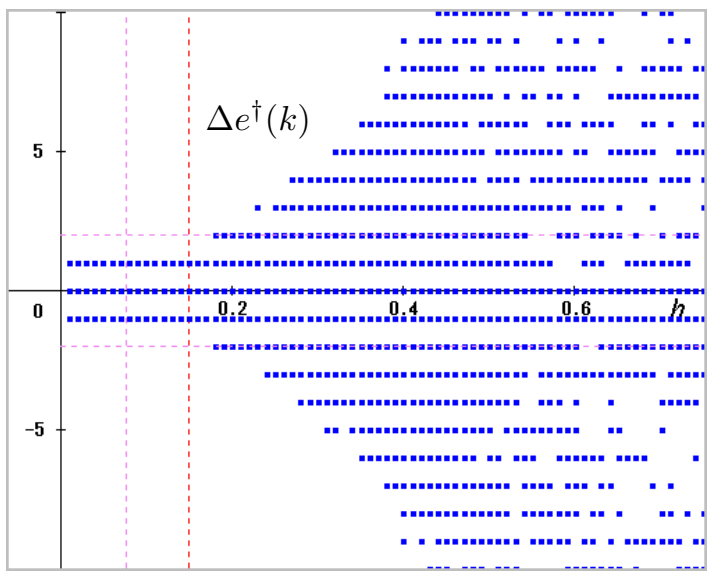

Fig. 15. Backward difference $\Delta e^{\dagger}(k)$ vs. sampling period $h$ for $r=2.0$.

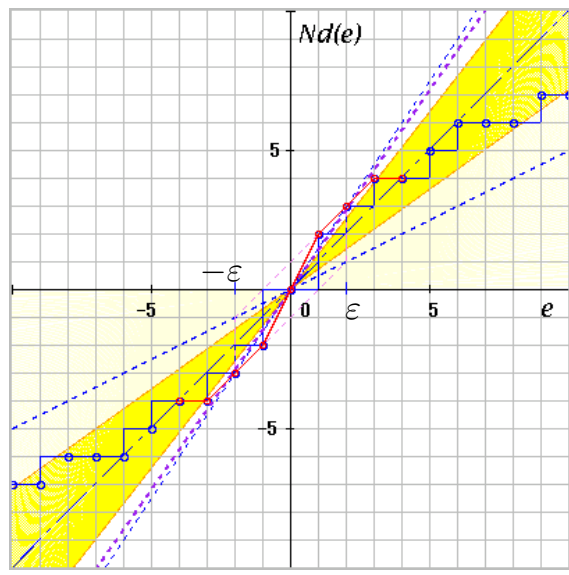

Fig. 16. Discretized nonlinear characteristic and stable sector for Example

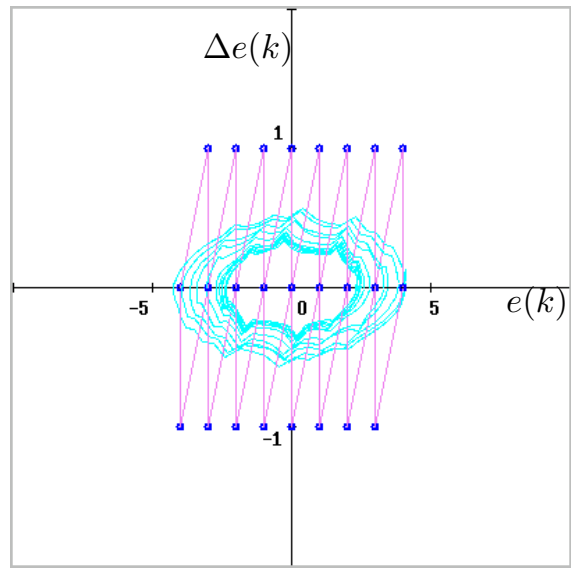

Fig. 17. Phase trace $(e(k), \Delta e(k))$.

can be determined as $[0.5,1.5]$ as shown in Example 1. Also in this example, the sampling period is chosen as $h=0.077$. The max-min value can be calculated as follows:

$$
\max _{q} \eta\left(q, \omega_{0}\right)=\eta\left(q_{0}, \omega_{0}\right)=0.285
$$

when the nominal gain $K=1.0$. Hence, inequalities (29) and (33) are given by $\alpha<0.285$, and the stable area is determined as $[0.715,1.285]$. This sector does not contain the above area $[0.5,1.5]$ as shown in Fig. 16.

Incidentally, the stability region of control systems with linear gain $K$ is given as $0<K<1.5$ when the sampling period is $h=0.077$. Thus, the discrete nonlinear feedback system in question corresponds to a counter example of Aizerman's conjecture. In this case, a phase trace and a step response are shown in Fig. 17 and 18, respectively. The output sequences of nonlinear element $f(\cdot)$ and the sum of trapezoidal areas $\sigma(k)$ are calculated as shown in Fig. 19. Figure 20 shows $\Delta e^{\dagger}(k)$ when the sampling period $h$ increases from 0.01 to 0.75 . In this case, assumption (8) is satisfied for $h<0.12$. Of course, the valid region and chaotic behaviors will be dependent on the magnitude of input $r$.

\section{COnCluding Remarks}

In this paper, the stability of discrete-time and discretevalue (discretized) feedback systems was considered, and the 


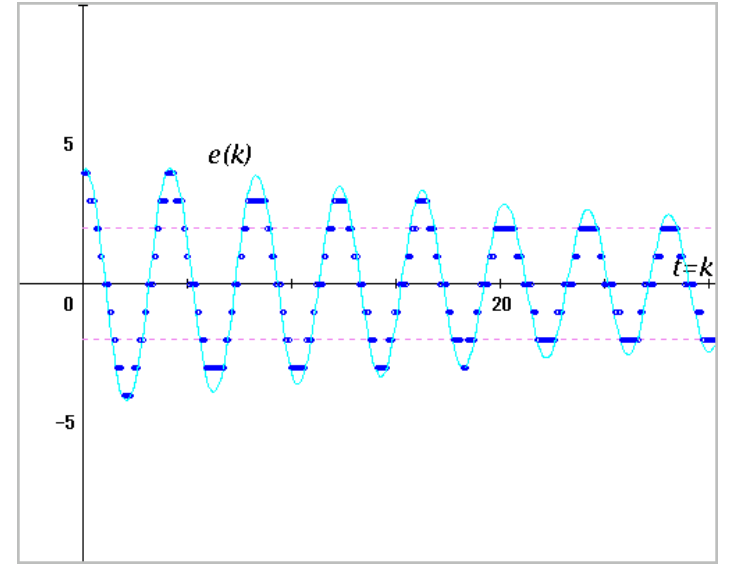

Fig. 18. Step responses $e(k), e^{\dagger}(k)$, when $h=0.077$.

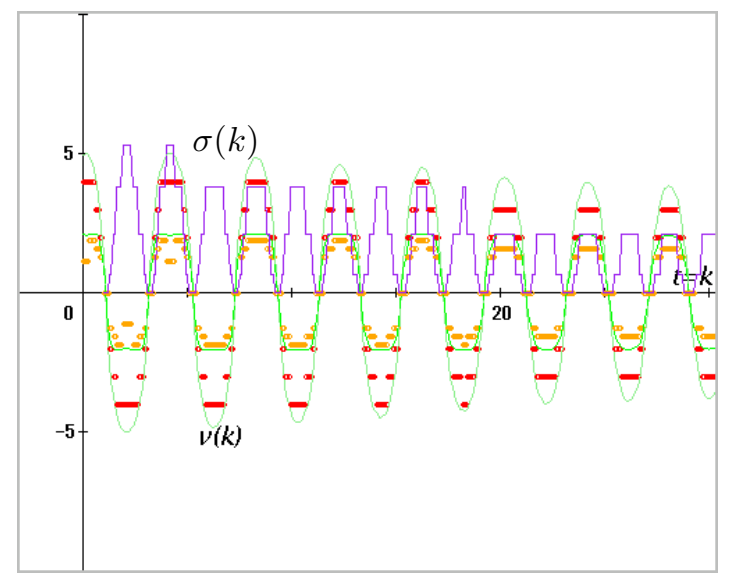

Fig. 19. Output signals $v(k), v^{\dagger}(k)$, and trapezoidal summation $\sigma(k)$ when $h=0.077$.

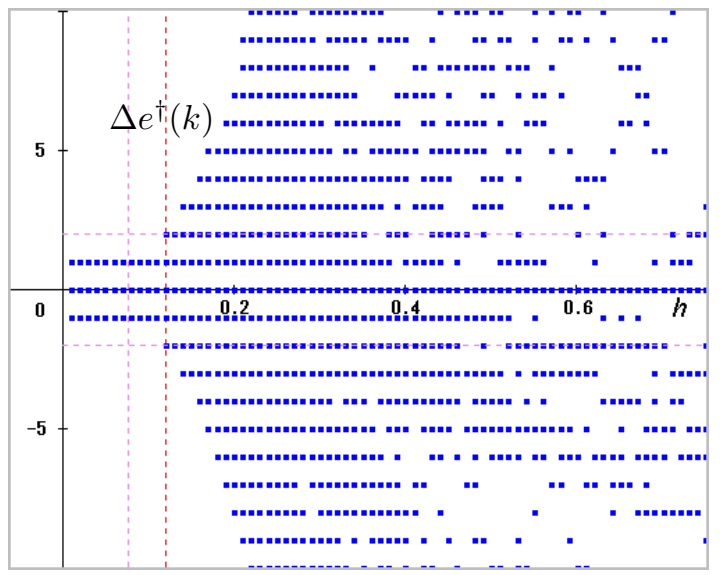

Fig. 20. Backward difference $\Delta e^{\dagger}(k)$ vs. sampling period $h$ for $r=4.0$. occurrence of (chaotic) oscillation was discussed in relation to the size of sampling period and the magnitude of input.

In this study, a non-conservative sufficient condition for the stability of discrete feedback systems is derived by applying the concept of robust stability in our previous paper [13]. The stability condition is, however, not satisfied for the entire area of the input of nonlinearity $N_{d}(e)$ because of the stepwise and point-to-point characteristic. Even if the response seems to be asymptotic, there may remain a fluctuation (a sustained oscillation in the discrete time) or an offset. Of course, a divergent response that reaches the sustained oscillation may occur. These responses will be typical nonlinear phenomena.

The theorems (and corollary) derived here should be considered as the (robust) stability condition in a global sense. In addition, it is valid based on an assumption in the relationship between the sampling period and the system dynamics. The author believes, however, that this result will be useful in designing a discrete (digital, packet transmission) control and communication system in practice.

\section{REFERENCES}

[1] N. Wiener, Cybernetics, (Control and Communication in the Animal and the Machine), The MIT press and J. Wiley \& Sons (Second ed.), 1961.

[2] N. Wiener, Time Series, (Extrapolation, Interpolation, and Smoothing of Stationary Time Series), The MIT press, 1977.

[3] H. W. Bode, Network Analysis and Feedback Amplifier Design, D. van Nostrand Co., Inc. (The Bell Telephone Laboratories Series), 1945.

[4] M. A. Aizerman and F. R. Gantmacher, Absolute Stability of Regulator Systems (Translated by E. Polak), Holden-Day, Inc., 1964.

[5] R. E. Kalman, "Nonlinear Aspects of Sampled-Data Control Systems", Proc. of the Symposium on Nonlinear Circuit Analysis, Vol. VI, pp. 273-313, 1956.

[6] S. P. Ragazzini and G. F. Franklin, Sample Data Control Systems, McGraw-Hill, 1958.

[7] E. I. Jury, Theory and Application of the z-Transform Method, J. Wiley \& Sons, 1964, revised by R. E. Krieger, 1973.

[8] R. E. Curry, Estimation and Control with Quantized Measurements, Cambridge, MIT Press, 1970.

[9] C. A. Desoer and M. Vidyasagar, Feedback System: Input-Output Properties, Academic Press, 1975.

[10] D. F. Delchamps, "Stabilizing a Linear System with Quantized State Feedback", IEEE Trans. on Automatic Control, Vol. 35, pp. 916-924, 1990.

[11] I. W. Sandberg, A Frequency Domain Condition for Stability of Feedback Systems Containing a Single Time-Varying Nonlinear Element, Bell Sys. Tech. J., Vol 43, pp. 1601-1608, 1964.

[12] G. Zames, On the Input-Output Stability of Nonlinear Time-Varying Feedback Systems, Pt. I and II, IEEE Trans. Automat. Contr. , AC-11, pp. 228-238, and pp. 465-477, 1966.

[13] Y. Okuyama and F. Takemori, "Robust Stability Analysis for SampledData Control Systems in a Frequency Domain", European Journal of Control, pp. 99-108, 2002.

[14] Y. Okuyama, "Robust Stability Analysis for Discretized Nonlinear Control Systems in a Global Sense", Proc. of the 2006 American Control Conference, Minneapolis, USA, pp. 2321-2326, 2006.

[15] Y. Okuyama, "Model-Reference Discretized PID Control and Robust Stabilization for Continuous Plants", Proc. of the 18th IFAC World Congress, Milano, Italy, pp. 5813-5818, 2011.

[16] Y. Okuyama, "Robust Stabilization of Discrete Model-Reference Control Systems on Integer Grid Coordinates", Proc. of the 2013 American Control Conference, Washington DC, USA, pp. 5613-5620, 2013.

[17] Y. Okuyama, Discrete Control Systems, Springer-Verlag, London, 2014. 